\title{
Représentations interculturelles dans le discours touristique des blogs de voyages français: une vision de l'Andalousie stéréotypée?
}

\section{Intercultural representataitons in the tourism discours of French travelling blogs: a stereotyped view of Andalusia?}

\author{
María LoReto CANTÓN RodríGUEZ \\ Universidad de Almería \\ lcanton@ual.es
}

\section{Resumen}

El desarrollo del discurso turístico en Internet ha cambiado la manera de diseñar y vender el producto turístico. En una decena de años hemos pasamos de hablar del turismo 2.0 al turismo 4.0. Este nuevo discurso integra el oral y el escrito para persuadir y convencer al turista internauta que ahora es emisor y receptor de un mismo producto. Esta nueva visión del Otro a partir de la práctica digital de los blogs de viaje será el objeto de esta contribución.

Ofreceremos, en primer lugar, una visión general teórica de este nuevo discurso turístico que ha renovado la manera de concebir la imagen de un país y sus identidades, a partir de la opinión de estos blogueros-turistas. Nuestro análisis se centrará, posteriormente, en la visión de Andalucía que estos viajeros dibujan diariamente en los blogs para verificar si la imagen que se traslada es engañosa, estereotipada o realza aspectos desconocidos de esta región para el turista potencial.

\section{Palabras clave}

Blog turístico, imagen, interculturalidad, Andalucía, viaje.

\begin{abstract}
The development of tourism discourse on the Internet has changed the way to design and sell the tourist product. In ten years, we have gone from talking about tourism 2.0 to tourism 4.0. This new discourse integrates oral and written discourse to persuade and convince the Internet tourist, who is now both transmitter and receiver of the same product. This new vision of the Other considered from the digital practice of travel blogs will be the subject of this contribution.

Firstly, we will offer a theoretical overview of this new tourism discourse, which has renewed the way of conceiving the image of a country and its identities, based on the opinion of these blogger-tourists. Our analysis will then focus on the vision of Andalusia drawn daily by these travelers in blogs, to verify whether the image conveyed is misleading, stereotyped or enhances unknown aspects of this region for the potential tourist.
\end{abstract}

\section{Key-words}

Tourism blog, image, interculturality, Andalusia, travel. 


\section{La communication touristique: le e-tourisme}

Le tourisme est l'une des principales sources de revenus tant en Espagne qu'en France. Avec la pandémie, les résultats du secteur ont entraîné une diminution du nombre de voyageurs français en Espagne. Savoir communiquer dans le secteur touristique suppose le fait de pouvoir le relancer. Les campagnes publicitaires institutionnelles et privées grâce à l'intégration des TIC (technologies de l'information et de la communication) ont beaucoup développé le secteur. Les acteurs du tourisme se réinventent pour attirer des consommateurs qui se rapprochent de plus en plus de ces nouvelles formes de communication afin de connaître les destinations de voyage et d'échanger des opinions. On pourrait, alors, définir la communication touristique avec le terme de e-commerce qui rassemble toutes les activités du secteur du tourisme qui se déroulent sur Internet. Philippe Viallon signale les éléments qui entrent dans cette définition:

[...] la place des réseaux sociaux numériques avant, pendant et après le voyage, la mobilité accrue des TIC (informations d'incitation ou d'attraction (pull ou push) partout et tout le temps) ou les nouvelles possibilités offertes par la technique (réalité augmentée, puce RFID, Web 3.0...) contribuent également à bouleverser les formes de communication du tourisme. (2018: 129)

Ces nouveautés provoquent un changement vis-à-vis des touristes qui deviennent émetteur et récepteur du produit. Ils sont appelés touristonautes, des personnes complètement intégrées dans le nouveau système numérique. Ils participent de ce processus, ils sont acteurs, producteurs et consommateurs de ce produit qui circule dans les réseaux.

Dans ce sens, le cyber-tourisme se situe, du point de vu linguistique, dans un discours de vulgarisation scientifique qui arrive à tout public. C'est à travers la communication que le client pourra accéder à la connaissance du produit touristique. La communication touristique $^{1}$ est, alors, le processus à partir duquel l'organisation (publique ou privée) qui crée un produit, informe de la disponibilité de celui-ci au touriste potentiel (Feminia, 2011). Le touriste ou touristonaute reçoit l'information mais il peut aussi la gérer et interagir avec l'Autre numérique (site web d'un tour-opérateur, hôtel, agence ou webs institutionnels et, surtout les forums ou blogs) et participer d'une manière active dans les communautés des voyageurs.

Cela est possible grâce au développement du Web.2.0. 3.0 et, surtout, les progrès du Web 4.0 qui diffère des précédents à certains égards importants: la géolocalisation qui per-

1 Pour les professionnels du tourisme, ce terme est toujours lié au marketing mais nous croyons indispensable le soutien d'autres disciplines pour comprendre tout l'ensemble des valeurs que le tourisme doit transmettre au voyageur. Selon Viallon: "La communication touristique est un concept très fréquent chez les professionnels qui la conçoivent comme une partie du marketing touristique. En revanche, elle est peu développée dans le monde scientifique. La raison est que les deux concepts qui la constituent, la communication et le tourisme, sont encore largement en devenir et que leur conceptualisation n'est pas terminé" (2018: 130). 
met au touriste d'avoir des informations immédiates sur l'endroit où il se trouve, la facilité d'obtenir des réponses via son téléphone mobile et le développement des apps qui lui permettront de payer les services touristiques fournis.

En conséquence, pour qu'un site promotionnel touristique se développe dans les meilleures conditions il doit être conçu avec des experts de plusieurs disciplines dont le marketing publicitaire et l'analyse de discours. En 1999 a été publié le Manifeste de Cluetrain qui a révélé une prise de conscience de tous ces changements qui se produisaient dans les marchés, nécessaire dans la nouvelle ère du numérique. La première des quatre-vingt-quinze thèses du manifeste est la suivante: "les marchés deviennent des conversations entre les entreprises et les consommateurs potentiels". Dans ce sens, le marché du e-tourisme est l'un de meilleurs exemples et les nouvelles typologies discursives ${ }^{2}$ mettent en place les relations sociales entre les acteurs touristiques. C'est le cas des relations entre bloggeurs et touristes, objet de ces lignes, car les blogs sont devenus l'un des derniers phenomène sociales dans tous les domaines de la vie quotidienne.

Nous allons envisager cette relation dans les blogs de voyage en trois lignes fondamentales:

1. L'aspect cognitif: le fait de vendre la destination à travers l'image offerte.

2. L'aspect conatif pour donner envie de se déplacer et de réserver.

3. L'aspect affectif qui sert à séduire et fidéliser le touriste potentiel.

Sur cette définition de Mahéo et Hénaff, reposent ces trois aspects:

[...] le blogueur communique son expérience touristique, sous tous ces aspects (découverte de lieux, hébergements, loisirs, activités, restauration), sous une dimension à la fois informationnelle et affective vers un public qui lui est acquis et qui partage souvent ses goûts et ses pratiques[...]. (2018: 152)

En outre, le développement des moyens de communication provoque également un rapprochement avec des cultures qui étaient auparavant inconnues, difficiles d'accès et limitées à un groupe de touristes à haut niveau d'achat.

Notre travail examinera, donc, ces aspects culturels dans les blogs du domaine touristique.

2 Ces nouveaux genres du discours ont été étudiés par plusieurs théoriciens (Baider, F. M. Burger et D. Goutsos 2004, Maingueneau, D. 2007, Mourlhon-Dallies, F. 2008, Calvi, 2010). Dans ces typologies, dans le domaine du tourisme et selon Calvi (2010), les forums et les blogs appartiennent aux genres informels face aux genres institutionnels (sites touristiques officiels, catalogues..) ou les genres commerciaux (sites touristiques privés, brochures commerciales, etc.). 


\section{Les blogs touristiques}

Un blog appartient à une plateforme UGC (User Generated Content) de partage d'information (forums, sites communautaires, wikis, etc). Le mot Blog est une contraction du mot Weblog, une forme créée en 1999 par Peter Merhol ${ }^{3}$. Pour J. Lendrevie et J. Lévy (2012) un blog est "un site web sur lequel une ou plusieurs personnes s'expriment de façon libre [et]régulière, avec une structure de présentation [antéchronologique] de plus récent au plus ancien]". Pour le blog touristique, il s'agit d'un renouvellement de la forme de carnet de bord qu'on trouve dans les journaux de voyages ou dans les guides touristiques. Le blog apparaît dans un site Internet et permet à une personne d'exprimer son avis et ses impressions des endroits visités. Ce sont des hypertextes où les blogueurs produisent et diffusent l'image d'une destination. De cette manière l'expriment Oriane Deseilligny et Caroline Ange:

Les blogs sont en effet fondés sur la publication d'un carnet de bord en ligne et la constitution d'un espace de communication entre un scripteur et des lecteurs. Ils sont marqués en outre par la circulation des formes et des pratiques de réécriture de fragments relevant de prescriptions habituellement liées aux guides touristiques imprimés. (2011: 132)

Les principales caractéristiques des blogs du domaine du tourisme sont:

Ils peuvent transformer l'image de la destination touristique grâce à la confiance que les blogueurs apportent au potentiel touristique. Nous parlerons ainsi de la croyance touristique qui transmet le blogueur sur une destination. Il faut différentier entre les blogs institutionnels et les blogs privés qui peuvent appartenir aux maisons d'éditions spécialistes en voyage et, les blogs privés. La plupart des blogueurs se considèrent sincères dans leurs opinions même s'ils sont bloggeurs professionnels, personnels ou corporatistes. Viallon et Henneken-Lange parlent de l'importance de croire (ou non) les informations transmises par les blogueurs car ils s'éloignent parfois des informations officielles. Ils déclarent:

[...] à côté de cette partie officielle du Web, s'est développée la partie officieuse: celles de blogs et des forums où chaque touriste peut raconter son voyage, photos à l'appui, peut encourager ou dissuader son semblable de se rendre à tel ou tel endroit. (Viallon \& Henneken-Lange, 2012: 79)

3 Les dictionnaires français en ligne font référence à l'idée de carnet de bord ou bien journal personnel mais il ne parlent pas de l'interactivité du blog pour le concepteur et l'utilisateur. Quelques exemples sont:

Le Larousse: abréviation de l'anglais weblog. Carnet de bord sur le web.

L'Internaute: Type de site internet permettant à une personne d'exprimer ses avis et ses expressions.

Le Robert: journal personnel, chronique d'humeur sur Internet.

Dans cette dernière définition, il peut être surprenant l'idée de lier le blog à l'humeur. Dans le cas du tourisme on trouve ce sens, par exemple, dans le Guide du Routard, si connu en France. 
Le blogueur devient ainsi le leader de la communauté des voyageurs. Tous les blogs ont leurs liens vers Facebook, Twitter, Instagram... Le blogueur ne peut pas transmettre ses créations (billets) sans un lien direct avec ces communautés du Web: "les blogues n'existent pas sans les réseaux sociaux qui leur permettent de générer du flux et de lancer des alertes vers les suiveurs et les abonnés” (Mahéo et Hénaff, 2018: 159).

Après l'ouverture d'un blog avec un certain nombre d'adeptes importants, les blogueurs peuvent en profiter pour publier des livres et des photos.

Tel qu'un guide touristique, le blog présente normalement trois parties importantes et différenciées sur la destination touristique: une information de base avec les données les plus objectives, une information complémentaire, plus subjective, et des informations pratiques. C'est dans cette deuxième partie informative complémentaire qui se développe l'éthos du discours, qui va marquer le ton de l'énonciation selon Maingueneau comme "[...]une manière de dire qui renvoie à une manière de penser et qui mobilise le co-énonciateur" (2007: 70). C'est la manière dont le blogueur privé va présenter l'information en toute liberté, tout au contraire que les blogs qui appartiennent à des organismes institutionnels ou ceux de grands tour-opérateurs. Quant au pathos, le style le plus affecté du discours, il vise à produire un effet émotionnel sur les sentiments du touriste ${ }^{4}$.

\section{L'identité dans la communication touristique}

L'image offerte par le discours touristique est essentielle pour le potentiel client avant de réaliser son expérience du voyage. Les stéréotypes, clichés et la perception de l'Autre détermineront des images préconçues que le touriste potentiel appréhendra d'une ou autre manière face à cette expérience.

Cela nous conduit également à réfléchir sur le concept de culture et d'interculturalité pour intégrer la vision de l'Autre à cette image. En 1982, l'UNESCO a défini la culture dans sa Déclaration de Mexico sur les politiques culturelles, comme suit:

La culture, dans son sens le plus large, est considérée comme l'ensemble des traits distinctifs, spirituels et matériels, intellectuels et affectifs, qui caractérisent une société ou un groupe social. Elle englobe, outre les arts et les lettres, les modes de vie, les droits fondamentaux de l'être humain, les systèmes de valeurs, les traditions et les croyances.

Le même organisme définissait interculturelle comme une notion "qui renvoie à l'existence et à l'interaction équitable de diverses cultures ainsi qu'à la possibilité de générer des expressions culturelles partagées par le dialogue et le respect mutuel" (2005).

4 Philippe Viallon propose une définition des trois concepts aristotéliens appliquée au discours touristique publicitaire: “[...] l'ethos correspond à l'image que l'émetteur donne de lui-même à travers son discours et renvoie à sa crédibilité et la confiance qu'il doit éveiller auprès du récepteur; le logos couvre l'argumentation mise en place et les stratégies afférentes; le pathos renvoie aux sentiments mobilisés et à tout l'affect de la communication" (Viallon, 2013: 3). 
Le tourisme est la principale activité pour la pratique de la culture et de l'interculturel car cette activité encourage l'échange d'opinions et d'activités parmi des personnes de la même culture ou de cultures différentes. À partir de la connaissance d'éléments locaux objectifs, comme la participation aux coutumes d'un pays, le touriste est plongé dans une culture différente qui lui permettra de s'enrichir et de participer activement à la connaissance de 1'Autre pour réaffirmer ses convictions ou les changer après son expérience touristique ${ }^{5}$. En plus, le tourisme, est une activité d'entreprise et économique. Dans un travail récent, Odile Challe (2020) s'interroge sur l'interculturalité dans le monde des entreprises. Tous ces aspects, qu'elle signale, sont importants pour les acteurs du domaine du tourisme. Elle affirme:

Le management interculturel a sans doute pour objet prioritaire d'améliorer ces interactions en milieu professionnel, convoquant non seulement la gestion mais la sociologie, la psychologie de l'individu et du groupe, l'anthropologie (qui étudie l'être humain), l'ethnologie (qui étudie la société) et la linguistique, parmi d'autres disciplines concordantes, puisque les comportements de chacun avec sa culture relèvent de la communication de nature intersubjective étudiée en sciences du langage. (Challe, 2020: 119)

Ce management est lié, aussi, au concept de stéréotype. Pour la révision de stéréoty$p e^{6}$, nous signalons, d'abord, les définitions de deux dictionnaires en ligne: Pour le dictionnaire Larousse, le stéréotype est:

Larousse: Caractérisation symbolique et schématique d'un groupe qui s'appuie sur des attentes et des jugements de routine.

Le Robert: Opinion toute faite réduisant les particularités.

Stéréotypes culturels: préjugés répandus.

Nous voudrions noter la différenciation pour le stéréotype culturel qui signale la deuxième définition de Le Robert. Pour ce qui nous concerne, ce sont ces idées par rapport au groupe qui sont transmises pour le touriste. De ce fait, la définition de Amossy et Herscheberg vient renforcer cette idée ${ }^{7}$ :

[...] l'image que se fait l'individu de lui-même est également médiatisée par son appartenance à un ou plusieurs groupes. [...] Les représentations collectives nécessairement sommaires qui s'attachent à chaque catégorie ont donc un impact considérable sur l'identité sociale. (Amossy \& Herscheberg, 1997: 32)

5 Nous suggérons la lecture de l'article de López Santiago (2020) et sa révision des termes culture et interculturalité proposée pour le domaine du tourisme.

6 Parfois, on parle aussi des clichés, terme qui a un rapport avec le mot stéréotype dans son origine typographique mais il reste plus figé dans le temps qui se répète sous la même forme (Amossy \& Herschberg, 1997).

7 En 1980, Louis Marie Morfauz définissait aussi le stéréotype comme: "images préconçues et figées, sommaires et tranchés, des choses et des êtres que se fait l'individu sous l'influence de son milieu social [...] et qui déterminent à un plus ou moins degré nos manières de penser, de sentir et d'agir" (1980: 34). 
Par ailleurs, l'image stéréotypée d'une destination touristique ne doit pas fournir une vision négative de celle-ci. On va examiner comment le marketing touristique profite des stéréotypes pour vendre, but final de la promotion des endroits. C'est important de mesurer à quel point cette vision change l'avis du consommateur à travers les blogs car selon Amossy et Herschberg:

Le stéréotype schématise et catégorise: mais ces démarches sont indispensables à la cognition, même si elles entraînent une simplification et une généralisation parfois excessives. Nous avons besoin de rapporter ce que nous voyons à ces modèles pour pouvoir comprendre le monde, faire des prévisions et régler nos conduites. (1997: 28)

Les représentations individuelles ou collectives, dans le domaine du tourisme, peuvent se transformer après l'expérience du voyage. À partir des expériences partagées dans les blogs, le touriste aura des références avant, durant et après son voyage. L'Autre devient, une attraction touristique, inséparable de ces visions. Selon Bugnot: "Los nuevos canales de distribución integrados en su difusión, las nuevas tecnologías como Internet representan un canal global propicio a la difusión de ciertas isotopías” (2009a: 60).

Le concept du stéréotype s'accompagne, dans l'analyse du discours, de celui de l'expérience touristique, forme auctoriale qui figure dans les blogs des sites spécifiques que nous analyserons. L'auteur devient aussi lecteur car les touristes sont invités à prendre la parole et son rôle est de médiatiser l'information. Conséquemment, il peut donner une autre vision de l'Autre. "Cette forme d'auctorialité renvoie tout entière à une énonciation de soi comme le montre la présence récurrente de la première personne" (Vergopoulos, Hécate \& Flon, 2012: 5).

Pour corollaire, il faut tenir compte aussi des imaginaires touristiques qui représentent les visions du monde, des topoïs spécifiques à travers la vison de la personne ou des groupes sociaux. L'imaginaire est l'ensemble de croyances et des images autour d'une activité, un espace, une période ou une personne (ou société) dans un moment donné. Les blogs touristiques témoignent de cette vision partagée (Hiernaux-Nicolas, 2002).

\section{L'Andalousie dans les blogs de voyages français}

La pandémie a fortement affecté le secteur du tourisme en Espagne. En 2020, ce pays a accueilli seulement 19 millions de touristes étrangers, soit 77\% de moins qu'en 2019. Les Français ont représenté le premier groupe de touristes dans le pays, avec 3,8 millions de visiteurs, malgré une chute de $65 \%$ sur un an, selon l'Institut national de statistique d'Espagne (INE). L'afflux de voyageurs français en Espagne avait surtout augmenté à partir de l'année 2008 et il s'est stabilisé au cours de la dernière décennie autour d'un peu plus de 11 millions de touristes. 


\begin{tabular}{|c|c|c|c|c|c|}
\hline \multicolumn{3}{|c|}{ Principales destinos en España de este mercado } & \multicolumn{3}{|c|}{ Ppales mercados extranjeros Andalucia } \\
\hline TOP 5 o6sTINOS - 2019 & Perroctadisnes & xis & TOP 5 OESGEN 2019 & Fernoct & $x y /$ totel \\
\hline Cataluña & 6.519 .730 & $36,1 \%$ & Reino Unido & 7.032 .768 & $24,0 \%$ \\
\hline Canarias & 2.878 .829 & $15,9 \%$ & Alemania & 3.750 .053 & $12,8 \%$ \\
\hline Balears, llies & 2.873 .306 & $15,9 \%$ & Francia & 2.526 .136 & $8,6 \%$ \\
\hline Andalucia & 2.526 .137 & $14,0 \%$ & Holanda & 1.427 .007 & $4,9 \%$ \\
\hline Madrid, Comunidad de & 828.926 & $4,6 \%$ & Italia & 1.328 .268 & $4,5 \%$ \\
\hline \multicolumn{3}{|c|}{ Fucnte: incuesta Ooupacila Hotecra. NE } & \multicolumn{3}{|c|}{ 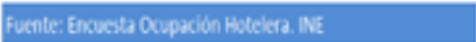 } \\
\hline
\end{tabular}

Figure 1

L'été 2020, l'Espagne avait lancé la campagne publicitaire L'Espagne vous attend pour essayer d'attirer les touristes après la longue période d'isolement. Cette année, avec Vous méritez l'Espagne (2021), le pays vise à renforcer le positionnement de l'Espagne comme principale destination de vacances des principaux marchés émetteurs (Royaume-Uni, France, Allemagne, Italie, Belgique, Suède, Pays-Bas et Pologne) et répond à la situation exceptionnelle causée par la pandémie ${ }^{8}$. Cette campagne essaie d'attirer le touriste international pour lui pousser à voyager et, surtout, le faire en dehors de son pays. Un grand groupe de ces touristes français véhiculent l'image de l'Espagne à travers les blogs touristiques'.

Après les îles (Baléares et Canaries) et la Catalogne, par proximité; l'Andalousie est l'une des régions des plus visitées par le tourisme français (Figure 1). L'objectif de ce travail est d'analyser l'image de l'Andalousie à travers les blogs des touristes français ${ }^{10}$. Il s'agit de trouver les éléments descriptifs pour examiner les stéréotypes dans le discours touristique de blogueurs selon les définitions révisées dans notre chapitre théorique.

D'ailleurs, notre étude vise à réaliser une analyse de l'image de cette région sur la base de thèmes d'identité et de stéréotypes. Pour cela, nous étudierons le texte et les éléments iconographiques présentés par les photographies que les blogueurs choisissent. Nous voulons également utiliser le modèle de marketing classique A.I.D.A appliqué aux blogs touristiques. Ce modèle se concrétise en quatre phases:

8 L'image d'Espagne a été renforcée par les campagnes des institutions publiques (le portail officiel du tourisme en Espagne (spain.info/es/), la Fundación Carolina, le Instituto Cervantes, et la Sociedad Estatal para la Acción Cultural Exterior o el Instituto Español de Comercio Exterior (ICEX). Voir aussi les études réalisées par Noya (2002). D'un point de vue de la France, on pourrait citer comme étude comparative, celle du Real Instituto Elcano (en Espagne) et la Asociación Diálogo (en France) en 2013 et celle réalisée récemment (2018) par Joël Brémond.

9 Selon le Baromètre Opodo/Raffour Interactif (2019), 84\% des Français préparent en ligne leurs voyages et 60\% ont tout réservé sur le $\mathrm{Web}$.

10 Dans un travail récent (Cantón: 2020), nous avons réalisé une analyse de l'image de l'Espagne en général dans un classement de blogs. Notre objectif était de savoir si les blogs offraient une image différente à celle offerte par les outils les plus classiques de la communication touristique (guides, brochures, livres de voyages etc.). Notre étude a choisi le classement des blogs réalisé par l'entreprise Kolsquare, celui de la revue Cosmopolitain et le blog Tourmondiste (2018). La plupart des classements est suggéré par les propres sites des blogueurs qui cherchent, parmi les réseaux sociaux, ceux qui ont un plus grand nombre de visites ou d'abonnés. 
1. Attirer l'Attention

2. Susciter l'Intérêt

3. Provoquer le Désir

4. Inciter à l'Action

Les entreprises du monde du tourisme travaillent avec les impressions des voyages. Ces impressions sont objectives du point de vu des images réelles (histoire, monuments, culture) et d'autres images subjectives crées à partir les impressions des autres voyageurs (amis ou famille) mais aussi des lectures faites, des reportages visionnés, etc. Les blogueurs ajoutent leurs expériences vécues qu'il partagent 24/24 et qui reçoivent des commentaires d'autres blogueurs et d'autres touristes: le blog est un outil conversationnel (Desseilligny et Ange, 2011) mais cette conversation se produit par le texte et par les images. Par rapport aux images, "la photographie est le support qui véhicule un nombre d'éléments imagologiques, stéréotypes ou mythes qui se réactivent dans l'esprit du récepteur et amplifient la fonction appellative du message" (Bugnot, 2009b: 21).

Le choix du corpus de blogs, pour notre étude, a été réalisé à partir de différentes compilations que les blogueurs eux-mêmes suggèrent. Nous nous sommes donc penchés sur les classements les plus récents de sites Web d'un certain prestige en fonction de leur importance par nombre de visites. Ce choix n'est pas facile car, les principales classifications, bien qu'ils offrent comme destination touristique l'Espagne, l'Andalousie ne s'y trouve pas ${ }^{11}$. Pour notre classement, nous allons explorer les blogs suivants ${ }^{12}$ :
1. Blog Andha Luz
6. Maman voyage
2. Andaluciamia
7. Les rêves d'ailleurs
3. Andalousie.style
8. Wild Child
4. Un allersimplepour
9. G3-guides.com
5. Marguerite et troubadour
10. Lavalinda

\section{Blog Andha Luz: https://blog.andhaluz.com/}

Le premier (Figure 2) s'adresse directement à une agence de voyage et, malheureusement, l'onglet direct vers le blog est ancien et les liens ne marchent pas. La dernière entrée date de 2016. C'est l'un des problèmes des blogs: s'ils n'ont pas des partenaires, de la publicité ou des abonnés, le blogueur, abandonne son voyage.

11 Dans le classement de Kolsquare en 2019, d'autres bloggeurs ont aussi présenté leurs voyages en Andalousie. La vision de ces blogs (Alex Vizéo, Un sac sur le dos, Travel me happy, On day) complète celle que nous allons réaliser dans ce travail.

12 Le soulignement du texte à partir des exemples est à nous, ainsi que les zones colorées des images qui nous semblent particulièrement significatives. 


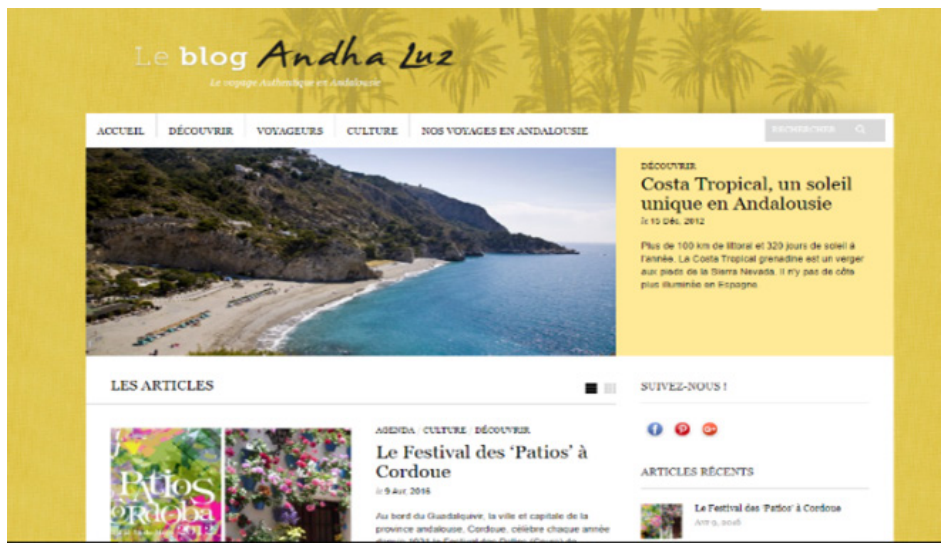

Figure 2

\section{Andaluciamia: https://www.andaluciamia.com/}

C'est un site basé sur la visite de l'Axarquia mais qui offre aussi d'autres voyages. Les onglets principaux sont: Découvrir/Activités/Préparer mon voyage/Agenda des Ferias/Blog Andalou. Dans la visite du blog (Figure 3), le blogueur fait référence à divers aspects de la culture andalouse. L'auteur affirme:

Ce blog est dédié à l'Andalousie, à travers ses ferias, ses traditions, son histoire, sa culture, son peuple, ses personnalités, sa gastronomie....Tout ce qui fait de cette terre un magnifique voyage à vivre intensément.

Découvrir l'Andalousie dans un paradis: l'Axarquia

Je vous propose, à la façon d'un voyage dans une Andalousie authentique et préservée, de découvrir l'Axarquia à travers ses villages blancs typiques, ses plages incroyables, ses traditions, ses habitants, sa faune et son héritage agricole toujours bien vivant. Une invitation au voyage. Tous ce qui est présenté est dans ce paradis, et donc dans un périmètre concentré, de $50 \mathrm{~km}$. maximum.

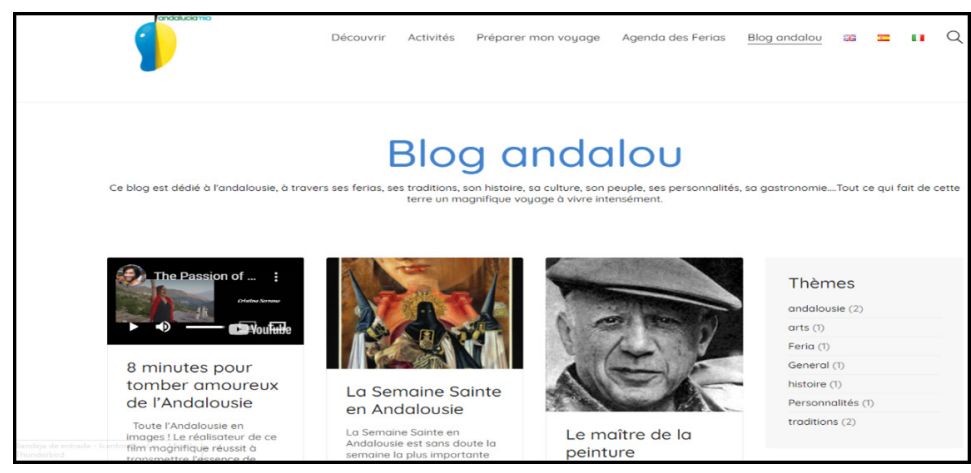

Figure 3 


\section{Andalousie.style: https://www.andalousie.style/}

Un autre blog dont le titre nous renvoie directement à l'Andolusie est celui-ci. Les blogueurs le présentent: “Andalousie.style c'est un blog de voyage sur cette merveilleuse région d'Espagne regroupant bons plans, conséils \& astuces pour vous guider lors de votre prochain voyage. [...] Notre style? Humoristique, documenté, nous avons comme seul objetif: partager nos passions" (Figure 4). Dans les onglets principaux, nous trouvons directement l'opinion des deux auteurs: Oriane et Angel.

Dans la section Villes, il est surprenant de constater le mélange entre les grandes villes andalouses historiquement visitées: Grenade, Cordoue, Séville; à côté d'autres villages tels que Ronda et Antequera qui appartiennent à la province de Malaga et par contre, le reste des villes andalouses ne se montrent pas sur le site. Cependant l'Alhambra dispose d'un lien direct pour obtenir des renseignements.

Quant au style du blog, ce sont les deux auteurs qui échangent leurs avis avec leur public: Sur la figure 5, on trouve l'avis d'Angel et d'Oriane.

Oriane dit: "Voilà ce qu'on espère te faire découvrir dans ce blog avec photos, supports, idées et bons plans pour préparer ton voyage ou simplement rêver". Pour Angel, "l'Andalousie est une région d'Espagne que j'affectionne tout particulièrement car elle me permet de me déconnecter très rapidement de la France".

Dans l'onglet: À faire ou pas, l'intention est de bien guider le potentiel voyageur pour qu'il profite bien de son séjour et lui faire comprendre qu'au-delà du tourisme culturel, il peut aussi se reposer:

Au départ vous avez envie de tout visiter, puis au bout de quelques jours d'un rythme infernal parisien, vous sentez que vous êtes en décalage avec le rythme local. Alors on visite çà ou pas?

Chère Oriane, la grande majorité des visiteurs qui séjournent en Andalousie aspirent à de la détente et de la découverte. Il faut donc probablement organiser des journées aérées de plages de repos, voire des journées $100 \%$ détente (piscine, tapas, etc...). Je dis çà, je dis rien.

Il est assez surprenant de ne pas trouver dans ce site des photos des plages de toute la côte andalouse. Dans un blog dont le nom est l'Andalousie, c'est juste une partie de cette grande région qui se montre. Cependant, c'est un bon site pour ce qu'on appelle les villages blancs: "En Andalousie les villages blancs sont une pure merveille d'authenticité". 
Anales de Filología Francesa, n. ${ }^{\circ}$ 29, 2021

REPRÉSENTATIONS INTERCULTURELLES DANS LE DiSCOURS TOURISTIQUE DES BLOGS DE VOYAGES...

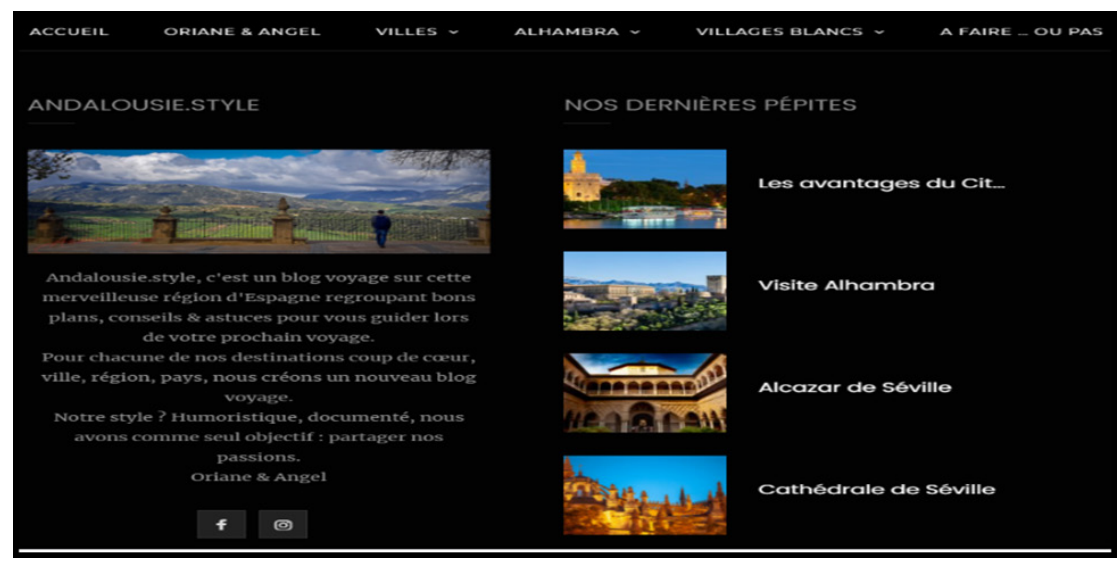

Figure 4

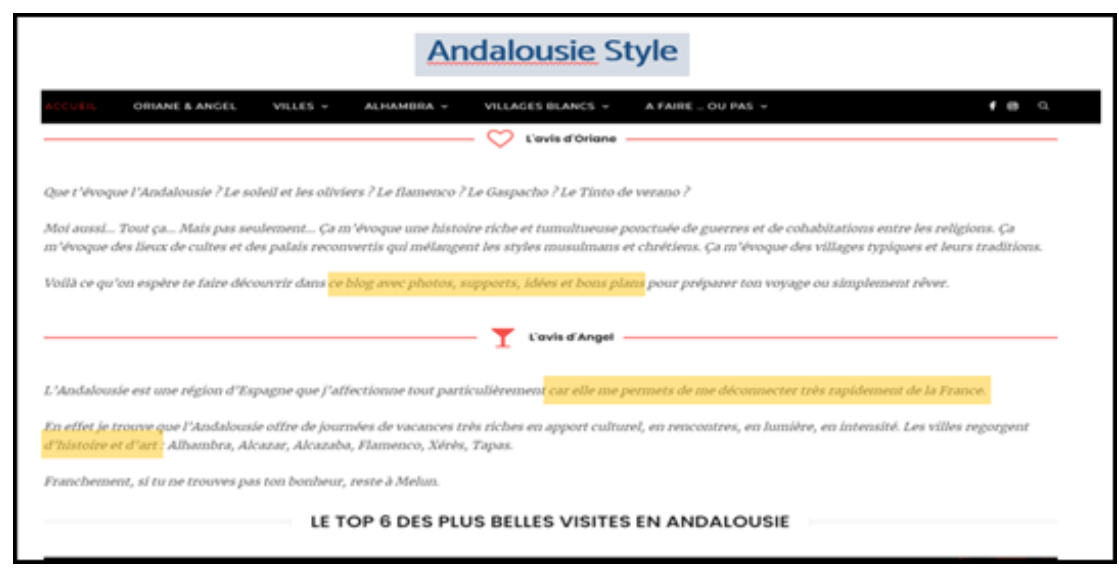

Figure 5

\section{Un aller-simple pour: https://unallersimplepour.com/}

Dans sa présentation, l'auteur souligne l'un des traits le plus important des blogs: “Le blog Un aller simple pour n'est pas un canal unidirectionnel. Votre histoire est aussi importante à raconter à nos yeux et aux yeux de tous.

C'est le seul blog où nous trouvons toutes les provinces de l'Andalousie et celui aussi avec un onglet pour parrainer le site: Devenir partenaire-annonceur. Il renvoie aussi à son site Facebook qui compte les abonnés. Même s'il y a très peu de billets à propos des villes, sur Découvrez mon Andalousie, on est surpris par certaines nouveautés dans les informations fournies. 
Anales de Filología Francesa, n. ${ }^{\circ}$ 29, 2021

MARÍA LORETO CANTÓN RODRÍGUEZ

- Vie pratique: l'auteur parle des écoles françaises dans la province de Malaga

- Le saviez-vous: ici, on trouve des histoires rares: Le Mannekin-Pis de Benalmádena

- Nature: un article sur les cigales en Andalousie surprend le lecteur

- Gastronomie: deux articles à propos des "espetos de sardinas" et "le tinto de verano".

Il s'agit d'un blog original mais avec peu d'information et des photographies. Peu de place pour les stéréotypes, dans ce cas, sauf pour la gastronomie.

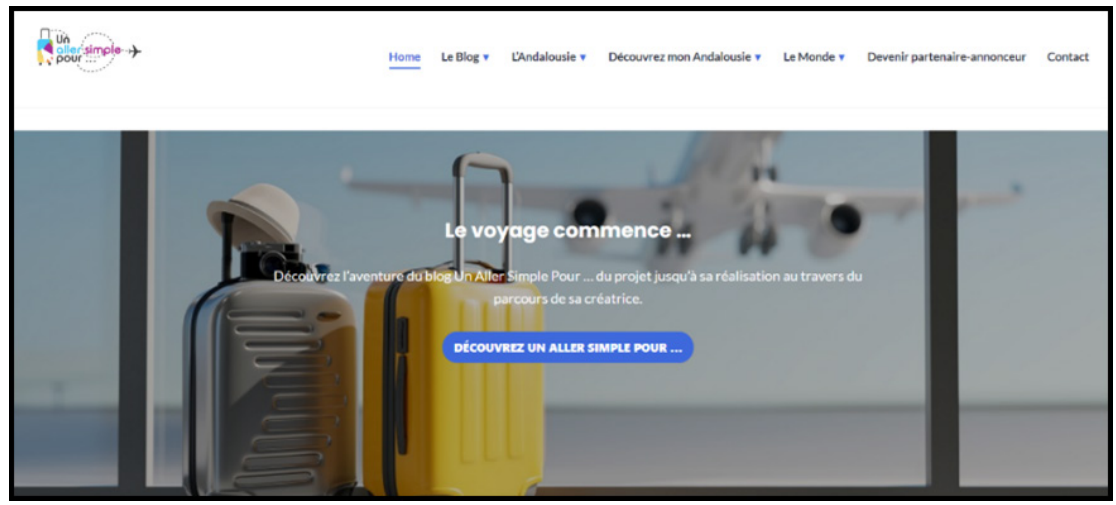

Figure 6

\section{Marguerite\& Troubadour: https://www.marguerite-et-troubadour.fr/}

C'est un blog général. Il faut fouiller un peu pour arriver à notre destination andalouse. Dans l'ongle Destinations, on arrive en Europe, après l'Espagne avec seulement deux endroits: L'Andalousie et la Grande Canarie.

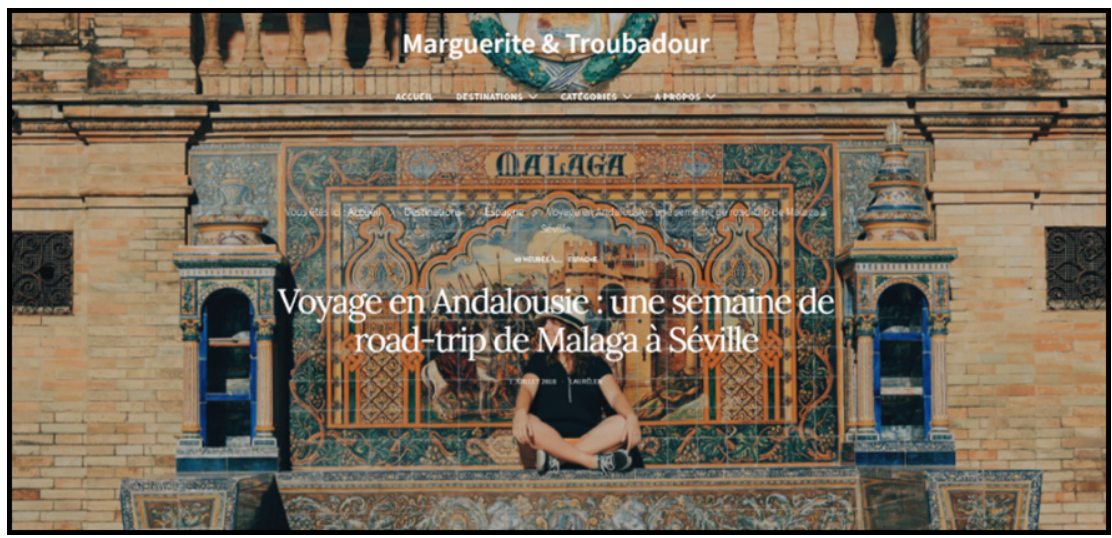

Figure 7 
Voilà la description faite de l'Andalousie, pour échapper de Paris:

Au mois de Mai, fuyant la grisaille parisienne, Célia et moi nous sommes envolées pour Malaga. Au programme: une semaine de road-trip en Andalousie de Malaga à Séville en passant par Grenade, Cordoue, Ronda mais aussi Nerja et Frigiliana sur la Costa del Sol. Je vous dresse le tableau: des villages blancs entourés de champs d'oliviers, des demeures ombragées et colorées articulées autour de sublimes patios et des influences maures à chaque coin de rue. L'Andalousie attire, l'Andalousie inspire. Et il ne nous en fallait pas plus pour être convaincues. Allez, c'est parti! Vous trouverez dans cet article tous mes conseils pour organiser votre voyage en Andalousie.

\section{Maman Voyage: https://www.mamanvoyage.com/tag/andalousie/}

C'est un blog de voyage en famille. Il est décrit dès le début: "le blog qui raconte tous nos voyages avec enfants depuis 2009! Nous avons déjà réalisé plus de 100 escapades en famille et un Tour du Monde en 2016 avec nos enfants (alias Ticoeur et Titpuce) qui ont maintenant 8 et 11 ans".

Dans ce blog les images prennent toute leur importance. C'est un blog très visuel où le texte est réduit (figure 8). Sur les images, une Andalousie stéréotypée de tapas, flamenco, corridas, et les principaux monuments historiques de Séville et Grenade. La figure 9 montre les opinions des visiteurs du blog qui ont bien aimé les photos. L'incitation au voyage est évidente: l'un des visiteurs veut acheter d'immédiat le billet pour partir: "j'hésite à cliquer sur l'aller retour trouvé fin septembre à $90 €$ pour y retourner".

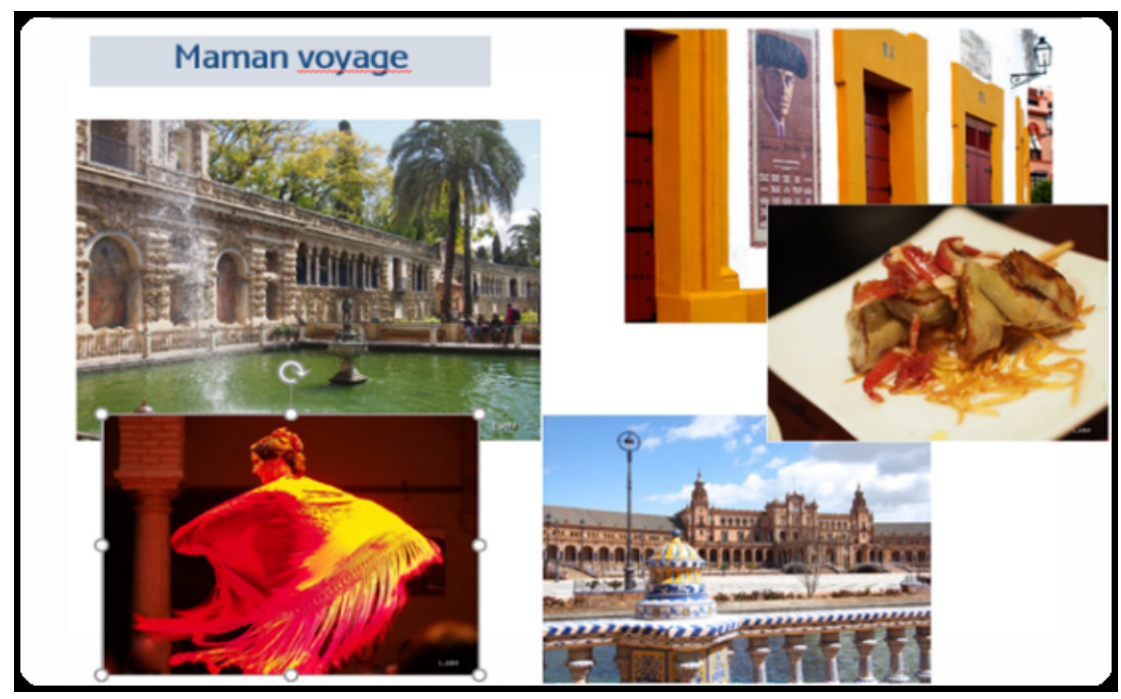

Figure 8 


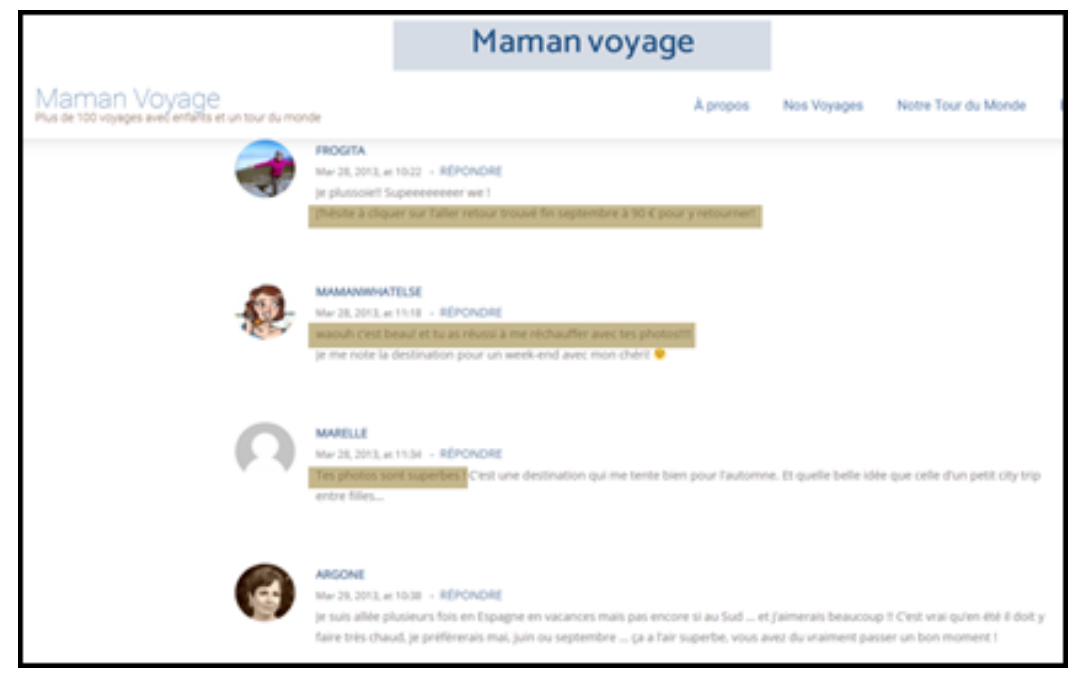

Figure 9

\section{Les rêves d'ailleurs: https://www.reverdailleurs.com/}

Il est défini comme "le blog de voyages sans gluten". C'est une présentation originale pour chercher une nouvelle typologie de touristes. La seule destination en Espagne est Séville. Il y a une présentation de Séville qui la compare à Barcelone, l'une des villes la plus visitée des Français: "Séville se situe au sud de l'Espagne, dans la région que l'on nomme 1'Andalousie. La vraie Espagne à mon sens. Barcelone ne l'est pas (ou plus vraiment). [...] En résumé, Barcelone c'est bien... Séville c'est mieux" (Figure 10).

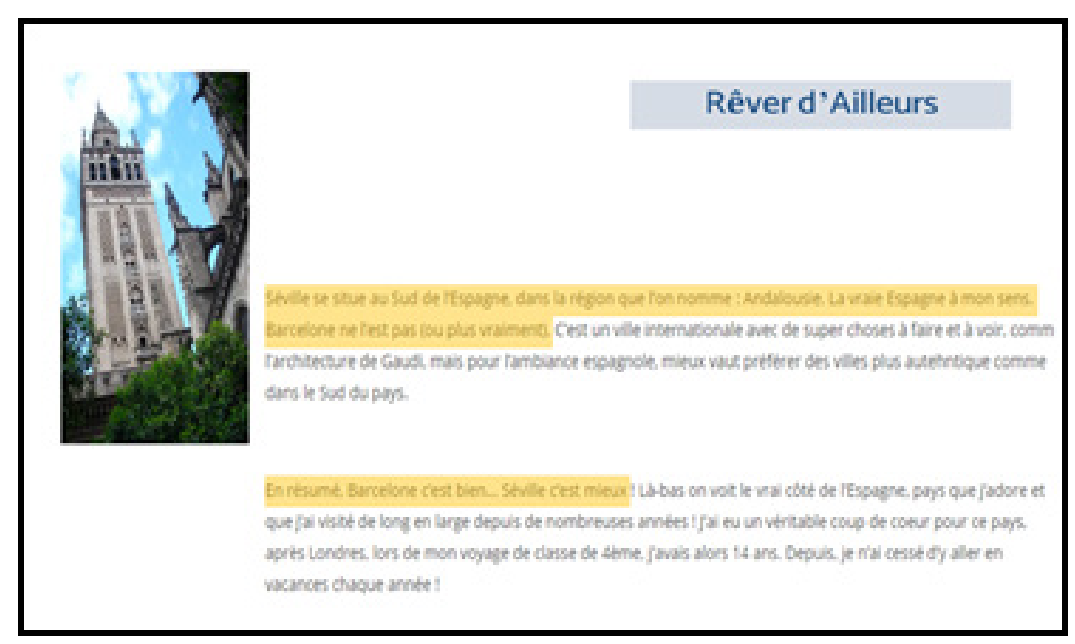

Figure 10 
Après, il explique les caractéristiques de la ville et des citoyens en termes de stéréotypes: le flamenco avec du lexique (culturemas) propres: "alegrías, bulerías, sevillanas ou encore les granadinas". L'auteur s'exprime en termes de sentiments humains. Après, il parle de la pratique de l'éventail à cause de la chaleur mais il ajoute: "je suis très adepte et je ne m’en sépare plus" (Figure 11).

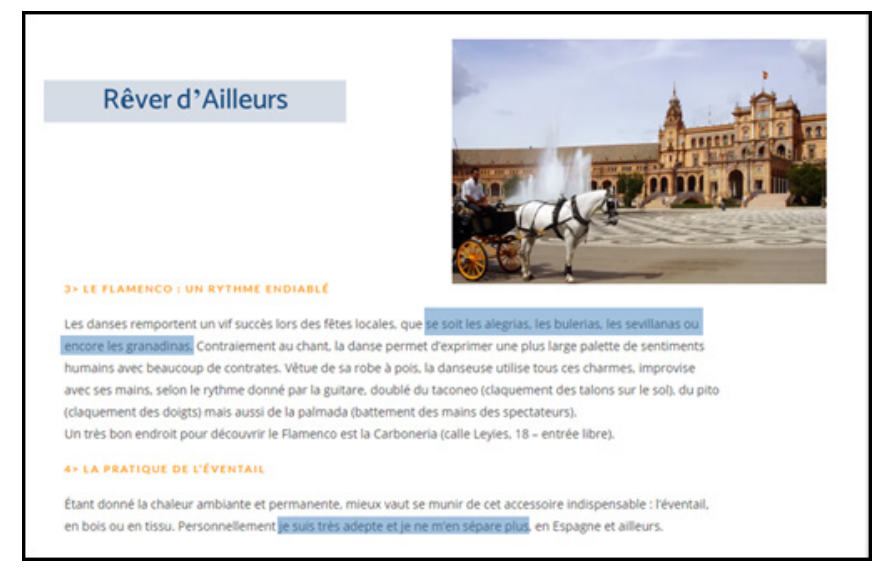

Figure 11

Il continue avec une réflexion sur la nuit sévillane et comment compléter le garderobe à Séville (Figure 12). De nouveau, une comparaison avec la France à propos des prix et des horaires des boutiques: "oui, oui la sieste est le sport national vous avez bien compris" (cliché répété sur l'Espagne en toute l'Europe).

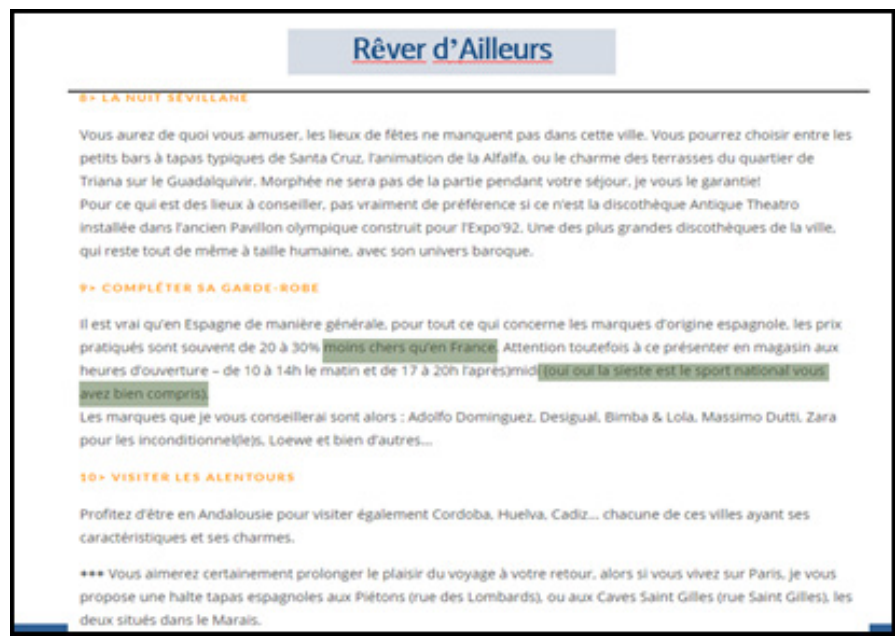

Figure 12 


\title{
8. Wild Child: Inspiration and Travel Blog: https://wild-child.fr/mon-itineraire-andalou/
}

La première manière d'attirer l'attention sur ce blog est la preséntation de l'auteur: “Salut, moi c'est AC: je suis libre, optimiste, curieuse prends des photos, médite, arpente le monde, je danse pendant des heures et je ris aux éclats. J'espère que vous apprécierez mon voyage... Pour en savoir plus, cliquez sur le dessin. Bienvenue sur mon blog!". Le "je”, du blog est une façon d'approcher les clients. Un langage jeune et proche pour attirer l'attention du lecteur.

Nous avons choisi ce blog parce que le seul voyage présenté pour l'Espagne c'est en Andalousie (figure 13) et parce que l'auteur la présente à partir de textes et d'images: Mon itinéraire andalou, et L'Andalousie en 15 clichés (sens photographique, Figure 14). Sur ces clichés, l'image de Grenade, Séville, Cordoue et les plages d'un village de la côte: Nerja. À nouveaux les images de l'Alhambra, les patios de Cordoue et les carreaux andalous, très typiques dans toute les photograhies de l'Andalousie et dans les souvenirs qu'on vend de la région.

En quelques lignes, l'auteur nous fait sentir l'esprit andalou à travers son histoire, ses monuments et, surtout ses paysages, et nous redirige vers ses photos et son réseau social.

\begin{abstract}
Au mois de juillet, j'ai remis mon backpack sur mes petites épaules pour partir quelques semaines à la découverte de l'Andalousie, au sud de l'Espagne. Cette région a été dominée par les Maures pendant de nombreux siècles, et en résulte une architecture unique, dont j'ai adoré photographier les détails.

Les rues pavées, les effluves de fleur d'oranger et d'huile d'olive, la morsure du soleil sur ma peau, la terre grise, orange, rouge, les notes de guitare qui dégringolent dans l'air au loin... les fleurs et les rivières, les palmiers et la mer. Cette ambiance magnifique m'a donné pour des mois de soleil
\end{abstract}

Pour plus de petites photos retraçant mon voyage, vous pouvez checker instagram, en cliquant sur la photo ci-dessous $(-)$

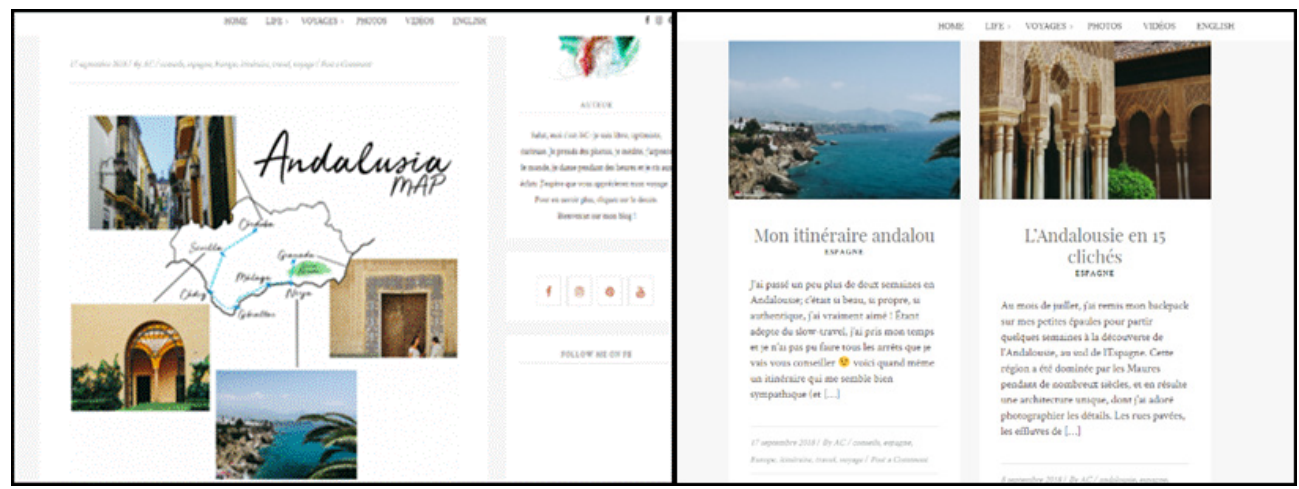

Figure 13

Figure 14 


\section{G.3 guides.com: https://g3-guides.com/fr/nous-sommes-guides-nature-en-andalousie/}

La présentation sur son site est: “G3-guides est une entreprise dédiée à l'écotourisme, au tourisme de nature, au tourisme slow, à la randonnée non sportive et à l'interprétation environnementale" (Figure 15).

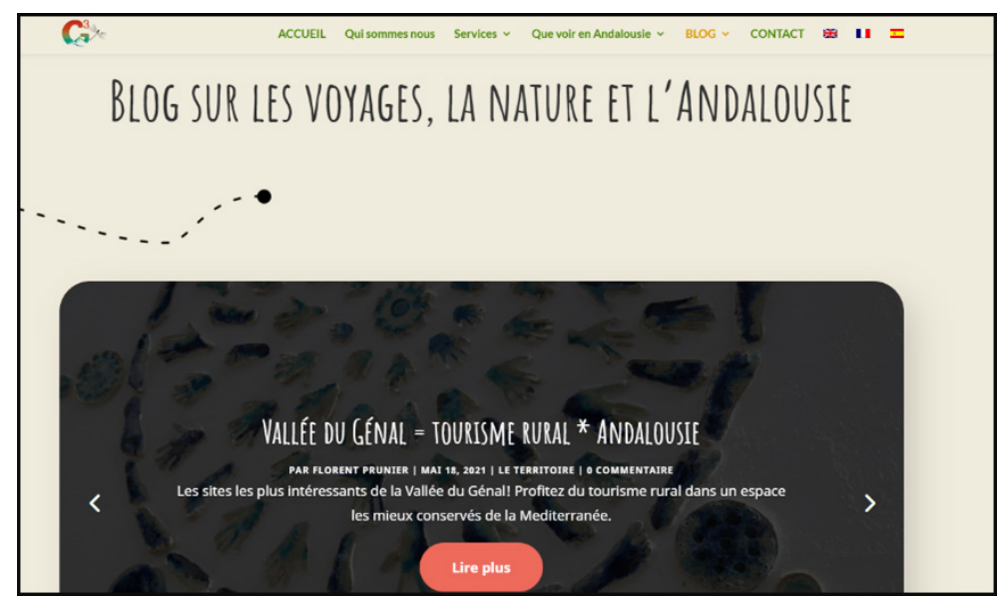

Figure 15

Dans ce cas, ce n'est pas un blog français mais espagnol qui offre la version française et anglaise dans son site et qui attire beaucoup l'attention des visiteurs par ses nouveautés dans le tourisme écologique.

Nous offrons nos services aux voyageurs, aux amoureux de la nature et aux familles à la recherche d'une expérience avec l'environnement naturel et le monde rural.

Notre engagement vise la cohérence entre notre action en faveur de l'environnement et une proposition de qualité. Par conséquent, notre éthique est basée sur le developpement durable et le respect de la culture locale.

C'est le blog avec une autre image de la région andalouse. La nature prend sa place et les excursions proposées sont totalement différentes. Un grand site trouvé pour les amateurs des activités en nature.

\section{Lovalinda: http://www.lovalinda.fr/lifestyle/voyages/europe/la-chic-aventure-andalouse/}

Une jeune qui abandonne sa vie pour devenir blogueuse: “J'avais une routine luxueuse en France pourtant j'ai tout plaqué pour une vie de bohème autour du monde" (Figure 16). 


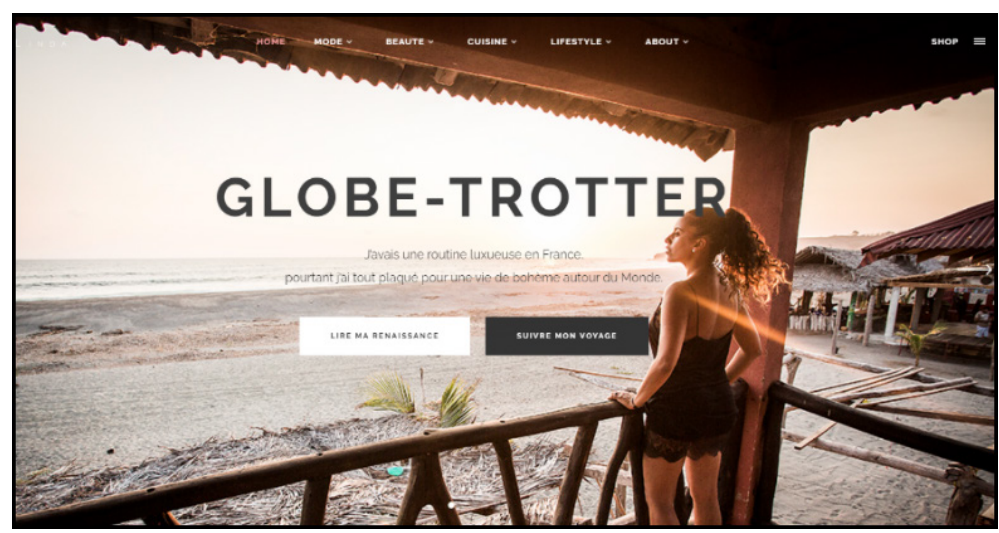

Figure 16

On trouve une manière très poétique de vendre la destination en utilisant les cinq sens de l'homme. Elle affirme dans sa conclusion:

On hume l'Andalousie quand ses parfums d'oliviers, de fleur d'oranger, de jasmin bordent les ruelles de ses quartiers.

On caresse l'Andalousie quand son soleil rebondit sur la beauté de ses plages de sable privées.

On écoute l'Andalousie quand sa guitare déchire la nuit embaumée.

On déguste l'Andalousie quand ses bars à tapas nous invitent à rentrer.

On lit l'Andalousie quand ses murs dessinent des arabesques multicolores estampillées au nom d'Allah et de son Protégé.

Mais surtout on la visite. Mon parcours chic et magique d'il y a deux ans, à jamais gravé, ne demande qu'à être copié. Je vous laisse, mon vol pour Barcelone décolle dans 1 heure. Bon premier week-end d'été!

Une Andalousie de parfums, des guitares, de tapas et l'empreinte arabe pour les monuments historiques.

\section{Conclusions}

Au fil de notre analyse, nous avons sélectionné un ensemble de blogs qui nous semblent pertinents pour l'étude de l'image de l'Andalousie en tenant compte des prémisses que nous avions posées dans notre approche théorique. Notre perspective s'est basée sur l'analyse des éléments du discours de promotion touristique à partir des images, des icônes d'une culture, des éléments intertextuels offerts par les voyageurs français et surtout, à partir des références textuelles propres à ce discours: adjectifs d'appellation, formules du moi personnalisé, appels au lecteur et, tout cela, dans un langage proche, propre des discours ordinaires, pour que le touriste potentiel interagit d'une manière familière. De plus, nous pouvons constater que le langage des répliques entre les auteurs des blogs et leurs adeptes ressemble à celui utilisé sur les réseaux sociaux, dans 
les messages de texte ou des applications de téléphones portables. On affirme aussi cette réciprocité d'information 24/24 comme l'une des caractéristiques de blogs avec la particularité du Web 4.0 de pouvoir réserver immédiatement, avec des liens directs à des sites touristiques andalous.

Quelles que puissent être les valeurs et la qualité des blogs, on peut conclure que c'est un moyen de plus en plus utilisé. Cela dit, quant aux blogs français qui nous proposent une vision des voyages à cette région, on peut conclure ce qui suit:

1. Il est difficile de trouver des blogs sur l'Andalousie ou d'autres avec des billets sur la région. L'accès se produit directement à certaines villes (Séville, Grenade, Cordoue), les plus connues et les plus stéréotypées: flamenco, tapas, arènes et leurs principaux monuments connus de tout le monde. La Giralda, l'Alhambra, les patios de Cordoue. Il existe alors une vision stéréotypée de l'imaginaire collectif.

2. Séville représente l'Andalousie parmi la plupart des blogs. L'Andalousie est une très grande région avec des diversités culturelles, linguistiques et sociales très marquées. La partie orientale ne s'identifie pas avec la partie occidentale andalouse. Il y a des villes avec très peu de références: Jaén, Huelva et Almeria sont les grandes inconnues.

3. Cependant, voilà encore une nouvelle vision de 1'Andalousie: la blogosphère touristique ouvre la voie à d'autres types de tourisme plus naturels qui concernent les espaces naturels, les parcs nationaux, les possibilités sportives...

4. Pour l'analyse textuelle, les images viennent renforcer la rapidité de lecture à laquelle les usagers des réseaux sociaux sont habitués. Il ne faut pas oublier l'âge moyenne des blogueurs et des leurs usagers. On peut assimiler les blogs aux réseaux sociaux qu'ils partagent. De plus en plus Instagram remplace Facebook. D'un côté les jeunes préfèrent l'image au texte et, d'autre, les usagers ont moins de temps et on peut dire que le proverbe une image vaut mille mots prend sa place.

5. Une certaine vision stéréotypée facilite l'objectif des blogueurs: vendre la destination touristique mais il serait convenable de réviser les textes qui appartiennent pour vérifier l'information et l'actualiser surtout dans les blogs institutionnels et les maisons d'édition.

Du point de vue du marketing touristique, il a fallu évaluer la qualité de l'interface. Selon le modèle A.I.D.A, les blogs nous ont proposé des solutions discursives et linguistiques dans les quatre aspects abordés.

\section{Attirer l'Attention:}

- Emploi d'une adjectivation positive et valorisante: Plages incroyables (2) ${ }^{13}$, les villages blancs pure merveille d'authénticité (2),

13 Les numéros renvoient aux blogs classés et analysés dans ce travail. 
magnifique voyage (2), sublimes patios (5), architecture unique (8), ambience magnifique (8), parcours chic et magique (10)

- Personnification des villes et des endroits touristiques: L'Andalousie me permets de me déconnecter, ses bars à tapas nous invitent à retourner (10) L'Andalousie attire, l'Andalousie inspire (5).

- Comparaisons avec des endroits connus par le lecteur: Franchement, si tu ne trouves pas le bonheur, reste à Melun.

Il a été offert en 1893 à la ville par Maria Luisa, duchesse de Montpensier, d'où il tire son nom.

- Emploi de photographies attirantes: des images parfaites avec des icônes typiques, en couleurs et, parfois, personnalisés avec les images des blogueurs:

Tes photos sont superbes! (6)

\section{Susciter l'Intérêt:}

- Des idées nouvelles: Notre engagement vise la cohérence entre notre action en faveur de l'environnement et une proposition de qualité (9)

- Avec des conseils pratiques pour le voyage: à faire ou pas (2), vie pratique, le saviez-vous (4), Vous trouverez dans cet article tous mes conseils pour organiser votre voyage en Andalousie (5)

- Nominalisation avec des slogans:

Le top de..., Les avantages de..., 10 bonnes raisons pour... Les incontournables... Un road trip... pour des connaissances rapides et des voyages à faire rapidement dans presque tous le blogs étudiés.

\section{Provoquer le Désir:}

- Perception de l'Autre: nous sommes semblables mais différents (comparaison avec des zones connues, personnages historiques et renommés...). Le maître de la peinture (à propos de Picasso) (2)

- Ils introduisent des vidéos et des photographies des stéréotypes (flamenco, tapas, arènes...).

- Des phrases attirantes: Ses traditionnelles tapas...pour donner l'eau à la bouche.

\section{Inciter à l'Action}

- Lien direct avec les centrales de réservation d'hébergement ou des billets ou aux réseaux sociaux des blogueurs: vous pouvez checker Instagram (8)

- Rétro-alimentation avec des expressions telles que: Je vous invite à..., je retournerai..., des impératifs: Découvrez mon Andalousie et les opinions d'autres bloggeurs connus avec des liens sur les divers réseaux sociaux et l'appel au 
Anales de Filología Francesa, n. ${ }^{\circ}$ 29, 2021

REPRÉSENTATIONS INTERCULTURELLES DANS LE DISCOURS TOURISTIQUE DES BLOGS DE VOYAGES...

lecteur, potentiel voyageur: Votre histoire est aussi importante à raconter à nos yeux et aux yeux de tous (4). J'espère que vous apprécierez mon voyage... Pour en savoir plus, cliquez sur le dessin (8).

En général, il s'agit des blogs avec des aspects attirants mais, dans notre cas d'étude, la recherche de billets sur l'Andalousie, devient parfois difficile et il faut prendre son temps pour naviguer sur le site. Ce sont des blogs qui répondent aux exigences de la communication touristique et qui utilisent des termes et des images stéréotypés dans le but d'attirer les touristes même si ce n'est à des fins commerciales comme c'est le cas des blogs privés analysés.

\section{Références Bibliographiques}

Amossy, Ruth \& Anne Herscheberg Pierrot. 1997. Stéréotypes et clichés. Paris, Éditions Nathan.

BAIDer, Fabienne, Burger, Marcel \& Dionysis Goutsos. 2004. La communication touristique. Approches discursives de l'identité et l'altérité. Paris, l'Harmattan.

Bugnot, Marie Ange. 2009a. "Estereotipia y localización en el discurso turístico" in Cédille. Revista electrónica de estudios franceses, $\mathrm{n}^{\circ} 5,56-80$. $<$ https://cedille.webs.ull.es/cinco/ bugnot.pdf $>$ [15/03/2021].

Bugnot, Marie Ange. 2009b. Le discours touristique ou la réactivation du locus amoenus. Granada, Comares (col. Interlingua).

Calvi, Maria Vittoria. 2010. "Los géneros discursivos en la lengua del turismo: una propuesta de clasificación" in Ibérica, $\mathrm{n}^{\circ}$ 19, 9-31: <http://www.aelfe.org/documents/01_19_Calvi. pdf $>[22 / 02 / 2021]$.

Cantón Rodríguez, María Loreto. 2020. "Nuevos espacios del discurso turístico: identidad e imágenes de España a partir de los blogs de turismo franceses" in Baynat Monreal, María Elena, Mercedes Eurrutia Cavero \& Cathy Sablé (eds.). Tic e Interculturalidad. Miradas cruzadas. Granada, Comares, 89-114 (col. Interlingua).

Challe, Odile. 2020. "L'interculturel. Une question de management international dans un monde connecté" in Baynat Monreal, María Elena, Mercedes Eurrutia Cavero \& Cathy Sablé (eds.). Tic e Interculturalidad. Miradas cruzadas. Granada, Comares, 115-134 (col. Interlingua).

Deseilligny, Oriane \& Caroline Ange. 2011. "Le maillage intertextuel des blogs de voyage" in Littérature et Communication, n 33. Paris, L'Harmattan. 131-140.

DufaYs, Jean-Louis. 1993 "Stéréotypes, lecture littéraire et postmodernisme " in Plantin, Christian (ed.) Lieux communs, topoï, stéreotypes, clichés. Paris, Kimé. 61-102.

Femenía Millet, Olga. 2011. La imagen de un destino turístico como herramienta de marketing. Málaga, Universidad de Málaga.

Hiernaux-Nicolas, Daniel, Allen Cordero \& Luisa Van Duynen Montinn. 2002. Imaginarios sociales y turismo sostenible. Costa Rica, Facultad Latinoamericana de Ciencias Sociales: <http://www.danielhiernaux.net/publicaciones/index2.php> [24/04/2021]. 
Anales de Filología Francesa, n. ${ }^{\circ}$ 29, 2021

MARÍA LORETO CANTÓN RODRÍGUEZ

Lendrevie, Jacques \& Julien LÉvy. 2012. Mercator. Théories et nouvelles pratiques du marketing. Paris, Dunod.

Levine, Rick, Christopher Locke, Doc Searls, \& David Weinberger. 1999. Le Manifeste de Cluetrain. Le manifeste des évidences: $<$ https://www.cluetrain.com/manifeste.html $>$ [24/04/2021].

López Santiago, Mercedes. 2020. "Hacia la interculturalidad a través de las TIC en el ámbito del turismo: Estudio contrastivo (Español-Francés)" in Baynat Monreal, María Elena, Mercedes Eurrutia Cavero \& Cathy Sablé (eds.). Tic e Interculturalidad. Miradas cruzadas. Granada, Comares, 223-248 (col. Interlingua).

MAHÉO, Claire \& Nolwenn HÉNAFF. 2018. “L'offre touristique portée par les blogs: entre information, prescription et communication commerciale" in Aquilina, Manuelle, Claire Mahéo \& Frédéric Pugnière-Saavedra (coord.). La communication touristique, vers des nouvelles interfaces? Laval, Presses Universitaires de Laval, 143-167.

Maingueneau, Dominique. 2007 [1998]. Analyser les textes de communication. Paris, Dunod.

Mourlhon-Dallies, Florence. 2008. Enseigner une langue à des fins professionnelles. Paris, Didier.

Morfaux, Louis Marie. 1980. Vocabulaire de la philosophie et des sciences humaines. Paris, Armand Colin.

UNESCO. 1982. "Déclaration de Mexico sur les politiques culturelles. Conférence mondiale sur les politiques culturelles": <file://D:/Datos/Descargas/D\%C3\%A9claration\%20de\%20 l'UNESCO $\% 20 \mathrm{de} \% 20$ mexico $\% 20$ sur $\% 201$ es $\% 20$ politiques $\% 20$ culturelles $\% 20(1)$.pdf> [15/04/2021].

UNESCO. 2005. "La Convention de 2005 sur la protection et la promotion de la diversité des expressions culturelles": <https://en.unesco.org/creativity/sites/creativity/files/2913_16 passport_web_f.pdf> [15/04/2021].

Vergopoulos, Hecate y Emilie Flon. 2012. “L'expérience touristique dans les guides: une subjectivité à lire, écrire et raconter" in Belgeo. Revue belge de Géographie. <http://journals. openedition.org/belgeo/7173> [15/03/2021].

Viallon, Philippe. 2013. "La communication touristique, une triple invention" in Mondes du tourisme, 7: <http://tourisme.revues.org/171> [10/02/2021].

Viallon, Philippe. 2018. "Le monde à portée de clic: la communication touristique en mutation" in Aquilina, Manuelle, Claire Mahéo \& Frédéric Pugnière-Saavedra (coord.). La communication touristique, vers des nouvelles interfaces? Laval, Presses Universitaires de Laval, 129-141.

Viallon, Philipe \& Sandrine HenneCKeR. 2012. "La croyance à l'épreuve d'internet: qui croire, le site ou le blog touristiques?" in Recherches en communication, $\mathrm{n}^{\mathrm{o}} 38$. $<\mathrm{https}$ ://core. ac.uk/download/pdf/288193968.pdf> [10/02/2021]. 
